\title{
8 Indeterminate Truth and Credences
}

\author{
Catrin Campbell-Moore
}

\subsection{Introduction}

Suppose you find yourself in the following unfortunate situation:

\section{PASSPORT}

If you have credence $\geq 0.5$ that you'll remember your passport, then when the time comes you'll end up forgetting it (you'll get on with other things).

And if you do not have credence $\geq 0.5$, then you will end up remembering it (you'll spend your time worrying about it).

And you know this about yourself. What should your credence be?

(Closely related to the Archer case from Joyce, 2018, or Basketball from Caie, 2013)

Suppose you adopt credence 0.2 that you'll remember your passport. Then you'll get on with other things and will forget your passport. And you know this. So then you should be certain that you'll forget your passport, i.e., adopt credence 1 . But were you to adopt credence 1 that you'll forget your passport, you would spend all your time worrying about it, and thus would remember it. And since you know this about yourself, this would recommend adopting credence value 0 . More generally, any credence value you assign will undermine itself in this sort of way.

Such scenarios have recently been discussed as a challenge for rationality. ${ }^{1} \mathrm{~A}$ rational opinion state should not undermine its own adoption. If it does, it cannot be relied on. And in a case like PASSPORT, every credence value is undermining so there seems to be no rational options.

We propose to parallel the kind of underminingness found in PASSPORT to that due to the liar sentence:

Liar: Liar is not true.

We might naturally reason about the liar sentence as follows: Is it true or not? Suppose it were true. Then since it says "Liar is not true", and Liar is 
true, we can conclude that it is false. Suppose it were not true. Then since it says "Liar is not true", we can conclude it is true. Reflecting on its truth value always results in a contradictory truth value; we might describe this as truth value assignments undermining themselves.

McGee $(1989,1990)$ argues that one should consider definite truth. Some sentences are definitely true, others are definitely false, but some, such as Liar, are indefinite. Inspired by the influential construction of Kripke (1975), in its supervaluational form, one can obtain an account of revision of definite truth value verdicts which allows for "fixed points". We might describe such fixed points as accounts of indefinite truth which are not undermining.

In this chapter, we propose to apply similar considerations to rational credence. The chapter starts with a summary of the account of truth in Section 8.2, and a presentation the classical account of credences and cases like PASSPORT in Section 8.3.

To account for these kinds of cases we propose allowing the notion of credence to be indeterminate, in particular, allowing that no definite credence value is assigned to PAssport. Our first question is how to model this (Section 8.4). One could just focus on questions such as whether one's credence in $\varphi$ is definitely equal to $r$ or definitely not equal to $r$, or neither. But we will suggest that it's more natural to directly consider the indeterminate credal state as a set of precise credence functions: its set of precisifications. So instead of focusing on definite judgements and considering the collection of precisifications as derivative, we directly work with the set of precisifications. This is a model of belief which is of independent interest in formal epistemology. Moreover, we can understand other supervaluational models by considerations of the precisifications, so by directly working with the set of precisifications, we can then also apply the results to other proposed models.

We then need to consider how to revise one's indeterminate credences, i.e., describe a supervaluational Kripkean jump. Section 8.5 proposes a very natural supervaluational jump, which revises a set of precisifications simply by revising each of the individual precisifications, we call this $\mathcal{R}$. But we will see that this can sometimes result in triviality.

To see how we might avoid this triviality, Section 8.6 returns to considering the supervaluational Kripkean jump for truth. For truth, one typically focuses simply on whether sentences are definitely true, definitely not true, or indefinite instead of focusing on the sets of precisifications themselves, even though it's this resultant set of precisifications that's important for defining the supervaluational Kripkean jump. We might also ask what happens when we focus on the set of precisifications themselves in the case of truth, and revise it by simply revising each of its members $(\mathcal{R})$. It too leads to triviality due to the McGee sentence. But the usual supervaluational Kripkean jump for truth (thought of as applying to an assignment of definite truth value verdicts) does not correspond to $\mathcal{R}$, but instead 
additional precisifications are added: those that agree on any definite truth value verdicts. This is what allows the usual account to avoid triviality.

We propose to apply this idea when we focus on sets of precisifcations themselves, as we did in the case of credences. Section 8.7 considers an alternative jump, closure $\circ \mathcal{R}$, which explicitly adds additional precisifications that are 'limits' of the precisifications obtained by revising each member of the set $(\mathcal{R})$. Formally, we take a topological closure. This will allow triviality to be avoided. It is moreover important to note that any focus on particular definite judgements for credences will not allow the triviality to be avoided in the way that it did for truth.

Section 8.8 proposes an account of when an imprecise credal state is non-undermining: when $\mathcal{R}(\mathbb{C}) \subseteq \mathbb{C} \subseteq \operatorname{closure}(\mathcal{R}(\mathbb{C}))$. Since there is always some credal state which is a fixed-point of closure $\circ \mathcal{R}$, there is always some credal state which is non-undermining in this sense. We thus propose that such credal states are candidates for being rational attitudes to adopt.

Section 8.9 demonstrates that the account we have provided is in fact very general and could apply to a whole range of target notions, one just needs to spell out a range of objects that play the role of the precisifications, and to describe how to revise each of these.

\subsection{Truth}

We first briefly present the usual account for truth.

\subsubsection{Classical, Precise Truth}

SETUP 8.2.1. Let $\mathcal{L}$ be a base language in which we have the ability to code sentences, for ease we might take this to be the language of Peano Arithmetic. Let $\mathcal{L}_{T}$ extend this with the addition of a unary pred-

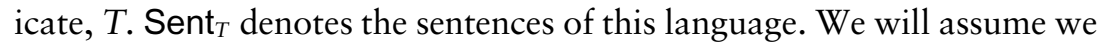
have a fixed model of our base language, which we assume is the standard model of arithmetic, denoted $\mathbb{N}$.

DefinITION 8.2.2. A precise interpretation of truth, $Q$, is given by a collection of sentences, i.e., $Q \in \mathcal{P}\left(\operatorname{Sent}_{T}\right)$. The collection of all precise interpretations of truth is AllPrecsT $=\mathcal{P}\left(\right.$ Sent $\left._{T}\right)$.

The collection $Q$ gives the collection of true sentences. We could equivalently think of it as assigning a truth value, true or not-true, to each sentence.

SETUP 8.2.3. ( $\mathbb{N}, Q$ ) refers to the classical model of $\mathcal{L}_{T}$ resulting from expanding the standard model of arithmetic for the base language, $\mathbb{N}$, with $Q$ providing the sentences whose codes are in the extension of the truth predicate. So we have $(\mathbb{N}, Q) \models T\ulcorner\varphi\urcorner$ iff $\varphi \in Q$. 
Definition 8.2.4. The Tarskian revision jump, $\tau$, is a function $\tau$ : AllPrecsT $\rightarrow$ AllPrecsT with $\tau(Q)$ given by

$$
\varphi \in \tau(Q) \text { iff }(\mathbb{N}, Q) \models \varphi .
$$

Note that we have $\varphi \in Q$ iff $T\ulcorner\varphi\urcorner \in \tau(Q)$.

The function $\tau$ can be understood as a stage of reflecting on the supposed truth values. To be materially adequate, an interpretation of truth should be a fixed point of $\tau$, i.e., $Q$ such that $Q=\tau(Q)$. But the liar paradox shows us that this is not possible because for the liar sentence, Liar, which is equivalent to $\neg T\ulcorner\operatorname{Liar}\urcorner$, we have that Liar $\in Q$ iff Liar $\notin \tau(Q)$. For an analogy with credences, we might say that every precise interpretation of truth is thus undermining.

\subsubsection{Definite Truth}

We now consider (in)definite truth (McGee, 1989, 1990). Some sentences are definitely true, for example ' $0=0$ ', some definitely not true, for example ' $0 \neq 0$ ', and some neither, for example Liar. A specification of which sentences are which is given by a definite verdict assignment:

DefinItion 8.2.5. A definite verdict assignment, $S$, is given by two sets of sentences, $S^{+}$and $S^{-}$.

$S^{+}$contains the sentences that are definitely true, and $S^{-}$the sentences that are definitely not true. ${ }^{2}$ Some sentences may be neither definitely true nor definitely not true. ${ }^{3}$

Associated with any definite verdict assignment is a collection of precise interpretations of truth, its "precisifications":

DefinItIon 8.2.6. $Q \in$ AllPrecsT is a precisification of $S=\left(S^{+}, S^{-}\right)$iff

- If $\varphi \in S^{+}$then $\varphi \in Q$.

- If $\varphi \in S^{-}$then $\varphi \notin Q$.

We call the collection of precisifications of $S, \operatorname{Precs}(S)$.

That is, $Q$ is a precisification if it agrees with the definite verdicts given by $S$ : any sentence that $S$ assigns as definitely true should be true in $Q$, and any sentence assigned as definitely not true should be not true in $Q$.

One might also consider adding "admissibility conditions" which restrict the precisifications to, for example, those that are maximally consistent. A more natural implementation of this in our framework is just to restrict AllPrecsT to such interpretations. For the purposes of this chapter, we will not consider such restrictions but it is easy to apply all our considerations with such restrictions, as we will mention in Section 8.9. 
We can also consider a set of precisifications giving rise to a definite verdict assignment:

Definition 8.2.7. Given a set of precisifications, $\mathbb{Q} \subseteq$ AllPrecsT, $\operatorname{Def}(\mathbb{Q})$ is a definite verdict assignment given by:

- $\varphi \in \operatorname{Def}(\mathbb{Q})^{+}$iff $\varphi \in Q$ for all $Q \in \mathbb{Q}$.

- $\varphi \in \operatorname{Def}(\mathbb{Q})^{-}$iff $\varphi \notin Q$ for all $Q \in \mathbb{Q}$.

That is, if $\varphi$ is determinately true in $\mathbb{Q}$, then it is assigned as definitely true by $\operatorname{Def}(\mathbb{Q})$, and if it is determinately not true in $\mathbb{Q}$ it is assigned as definitely not true by $\operatorname{Def}(\mathbb{Q})$.

The supervaluational Kripke jump revises a definite verdict assignment as follows:

DefinITION 8.2.8. $\Delta(S)$ is the definite verdict assignment given by:

- $\varphi \in \Delta(S)^{+}$iff $(\mathbb{N}, Q) \models \varphi$ for all $Q \in \operatorname{Precs}(S)$.

- $\varphi \in \Delta(S)^{-}$iff $(\mathbb{N}, Q) \vDash \varphi$ for all $Q \in \operatorname{Precs}(S)$.

Since $(\mathbb{N}, Q) \vDash \varphi$ iff $\varphi \in \tau(Q)$, we can give an alternative description of this: $\Delta(S)$ is the definite verdict assignment given by looking at the determinate judgements of the collection of precisifications $\tau(Q)$ for $Q \in \operatorname{Precs}(S)$. That's exactly what Def allowed us to state, so:

$$
\Delta(S)=\operatorname{Def}(\{\tau(Q) \mid Q \in \operatorname{Precs}(S)\}) .
$$

We can further simplify this by making another definition.

Definition 8.2.9. For a set of precisifications $\mathbb{Q} \subseteq$ AllPrecsT,

$$
\mathcal{R}_{\tau}(\mathbb{Q}):=\{\tau(Q) \mid Q \in \mathbb{Q}\} .
$$

The operation $\mathcal{R}_{\tau}$ just revises each member of the set in accordance with $\tau$, so $\{\tau(Q) \mid Q \in \operatorname{Precs}(S)\}=\mathcal{R}(\operatorname{Precs}(S))$. We then immediately have:

$$
\Delta(S)=\operatorname{Def}\left(\mathcal{R}_{\tau}(\operatorname{Precs}(S))\right) .
$$

We can use $\circ$ to denote concatenation, so write this as

$$
\Delta(S)=\operatorname{Def} \circ \mathcal{R}_{\tau} \circ \operatorname{Precs}(S) .
$$

We can describe this by the following procedure for obtaining $\Delta(S)$ :

(i) Starting with a definite verdict assignment, use Precs to move to the corresponding set of precisifications.

(ii) Revise each of the precise interpretations in the set according to $\tau$ (i.e., apply $\mathcal{R}_{\tau}$ ). 
(iii) Use Def to move from the resultant collection of revised precise interpretations to the definite verdicts.

There are fixed points of $\Delta$ : accounts of definite truth that are nonundermining. Moreover, there are non-trivial such fixed points.

\subsection{Precise Credences}

We now move to developing the analogous tools for the case of credences. We start with the classical, precise setting.

\subsubsection{Credence Functions}

We first start just by specifying the notion of credence function that we're working with in the classical setting.

SETUP 8.3.1. We start with a non-empty set of sentences, $\mathcal{A}$, which we call our agenda. ${ }^{4}$

This could be all sentences of a given language, but it can also be more restrictive, for example we might consider cases where we are only looking at your credence in a single sentence, so where $\mathcal{A}$ is a singleton. For example we might just be interested in the credence that you'll forget your passport, so $\mathcal{A}$ might just contain the sentence saying that you'll forget your passport. ${ }^{5}$

Definition 8.3.2. A credence function on an agenda $\mathcal{A}$ is a function, $c$, from $\mathcal{A}$ to $[0,1]$; i.e., it associates with each sentence in $\mathcal{A}$ a degree of belief, which is a real number between 0 and 1 inclusive. $\operatorname{Creds}_{\mathcal{A}}$ is the set of all credence functions, i.e., all functions from $\mathcal{A}$ to $[0,1]$. If $\mathcal{A}$ just contains a single sentence, a credence function can be thought of simply as a value in $[0,1]$ and we will call this a credence value.

It wouldn't affect our account if we restrict Creds $_{\mathcal{A}}$ to just those functions that are finitely-additive probabilities (or, more carefully, which are extendable to functions on a Boolean algebra which satisfy the axioms of finitelyadditive probability theory ${ }^{6}$ ), as this is still a compact space. However, we could not restrict attention to countably-additive probabilities. ${ }^{7}$

\subsubsection{Revision of Precise Credences}

The Tarskian revision jump, $\tau$, was a way of revising precise interpretations of truth. For credences, we will suppose we have a revision function as given, and our supervaluational account will be a general one that can apply to any given revision function. ${ }^{8}$ Formally:

Definttion 8.3.3. A revision function is a function, $\rho$, from $\operatorname{Creds}_{\mathcal{A}}$ to Creds $_{\mathcal{A}}$. 
A precise credence function is undermining if $\rho(c) \neq c$.

PASSPORT is a story which directly describes how one should revise one's credence in the target-proposition, that you'll remember your passport, under a step of reflection on the proposed credence value. If we let $\mathcal{A}$ simply contain this one proposition, then credence functions are just values $x \in[0,1]$, and the revision function that PASSPORT gives rise to is:

$$
\rho_{\text {PASSPORT }}(x)= \begin{cases}1 & x<0.5 \\ 0 & x \geq 0.5\end{cases}
$$

One can see that there are no fixed points of $\rho_{\text {PASSPRT }}$. That is, every precise credence function undermines its own adoption. In this sense PASSPORT can be related to the liar sentence: in both cases, all precise options are undermining.

There are other cases that also give rise to the same revision function as PASSPORT, for example:

\section{Bad NAvigator}

You've come to a crossroads and are wondering whether you need to turn left or right to get to your hotel. You know you're a really bad navigator. In particular, you believe that if you have credence $\geq 0.5$ that left is the way to your hotel, then it's actually right; and if not, then it's actually to the left. What should your credence be that it's actually right?

(Extremal version of an example in Egan and Elga, 2005)

In this case, the change in one's credence isn't due to the causal structure, but instead simply that the credence that one adopts affects the evidence that one has about the situation. But the same revision function describes this case. The same revision function would also arise when considering the following self-referential sentence:

CredLiar: Your credence in CredLiar is not $\geq 0.5$.

In this case, the revision is due to semantic features of the sentence.

You might also be uncertain about whether you are in a PASSPORT-like case:

\section{GolF}

You think there's a small chance, $1 \%$, that whether you'll be able to successfully get this hole-in-one is dependent on the credence you adopt in it in a PASSPORT-style way, i.e., where if you have credence $\geq 0.5$ then you'll fail, and if not then you'll succeed. But you're $99 \%$ sure that it's just a normal case and you have a $50 \%$ chance of success. 
This leads to the revision function

$$
\rho_{\text {GOLF }}(x)= \begin{cases}0.01 \times 1+0.99 \times 0.5=0.505 & x \geq 0.5 \\ 0.01 \times 0+0.99 \times 0.5=0.495 & x<0.5\end{cases}
$$

It also might be that one's credence doesn't directly provide evidence about the truth of the sentence, but instead it affects the chances. Consider, for example, the following scenario discussed by Greaves (2013):

\section{PROMOTION}

"Alice is up for promotion. Her boss, however, is a deeply insecure type: he is more likely to promote Alice if she comes across as lacking in confidence. Furthermore, Alice is useless at play-acting, so she will come across that way iff she really does have a low degree of belief that she's going to get the promotion. Specifically, the chance of her getting the promotion will be $1-x$, where $x$ is whatever degree of belief she chooses to have in the proposition $P$ that she will be promoted. What credence in $P$ is it epistemically rational for Alice to have?"

(Greaves, 2013, pp. 1-2)

(Moreover Greaves assumes "that the agent is aware of the specification of ... her case".) If Alice considers adopting credence 0.2 in $P$; then the chance of $P$ would be 0.8 , and she knows that, so that would recommend adopting credence 0.8. More generally, the description of this case directly provides us with the revision function

$$
\rho_{\text {Рвомотіо }}(x)=1-x \text {. }
$$

Unlike for the PASSPORT revision function, this function does have a fixed point, 0.5 .

All the cases we've seen so far are unusual cases. In normal cases, the credence one adopts provides no additional evidence about the situation at hand.

\section{RAIN}

The credence that you adopt that it is going to rain tomorrow provides no additional evidence about the likelihood of rain.

In this case, $\rho(x)=x$. More generally, in normal cases $\rho(c)=c$ for all $c$, or at least all $c$ which are probabilistic. ${ }^{9}$ And most theorising about rationality has focused on these "safe" cases.

This same revision function might also arise in a case where the credence one adopts does provide additional information: 
LEAP

The chance you'll successfully leap across this chasm is identical to your credence.

(Greaves, 2013, see also James, 1897)

We do not assume any further modelling of this revision function, we simply assume that any scenario gives rise to such a revision function. Any further modelling of $\rho$ would have allow for the range of cases mentioned so far. It has to allow for logical, causal and evidential impact (as in CredLiar, PASSPORT, and BAD NAVIGATOR), and this might go via chance (like Promotion) or be directly about the proposition (as in PASSPORT), or be associated with further uncertainty. Our account does not depend on any further specifics of the revision function, we can simply take it as an input to our account. The revision function should encode the idea of reflecting on one's credences, and we are adopting the idea that to be rational, a precise credence function should be a fixed point of this revision function..$^{10}$ Otherwise a credence function is undermining.

There are two suggestions for how one could include further modelling or explanation of the revision function.

Firstly, one might simply take $\rho(c)$ to be $c$ conditionalised on " $c$ is my credence function". If we assume that one's initial credence function satisfies certain other constraints of rationality such as deferring to chances (by satisfying the so-called Principal Principle) this should lead to the revision functions proposed. The idea of this is to result in notions like those of Joyce (2018) or Konek and Levinstein (2019), as opposed to the "consequentialist" recommendation notions of, for example, Pettigrew (2018) and Caie (2013). ${ }^{11}$

Alternatively, one might want to explicitly include a possible worlds structure in the modelling and then define $\rho$ using this. This is particularly natural for accounting for a language with sentences that can talk about the credence one has in that very sentence, i.e., cases like PrLiar. This kind of picture has commonly been used when developing accounts for languages with modal predicates (Halbach et al., 2003; Stern, 2015; Campbell-Moore, 2015; Halbach and Welch, 2009; Nicolai, 2018). Given a fixed possible world structure, with various worlds and probabilistic accessibility relations between them, we consider a precise interpretation to be given by an assignment of a credence function at each world: a credal-evaluation-function. We can then directly define the revision of a credal-evaluation-function by taking the weighted proportion of the accessible worlds where the sentence is evaluated as true when the initial credal-evaluation-function provides the interpretation of the credence function symbol at the various worlds. ${ }^{12}$

There are two reasons to focus simply on credence functions rather than using a possible world structure. Firstly, it is simpler and all our considerations will immediately apply to the more general setting (Section 8.9). 
Secondly, it is not directly obvious how to provide a possible worlds model for the cases like PROMOTION where the impact goes via chances. And insofar as it deals with PASSPORT or BAD NAVIGATOR it just treats them like CredLiar, for example, we wouldn't represent 'the hotel is to the left' as an atomic sentence, as would be most natural, but instead as a sentence that refers to itself. Whilst this leads to the right revision function, it does not seem to be the right analysis of the sentence itself. We thus find it valuable to not encode further modelling such as this, but to simply provide the account for any specified revision function; though in section 8.9 we note how our account immediately applies to the possible worlds setting.

\subsection{How to Model Indeterminate Credences}

In the case of truth we focused on definite truth value verdicts. When considering indeterminate credences, what should we think about? We suggest that we directly work with a set of precise credence functions, that is, we consider one's indeterminate credal state to be given by a non-empty set $\mathbb{C} \subseteq \mathrm{Creds}_{\mathcal{A}}$. The precise credence functions in the set will be called the 'precisifications' of the indeterminate credal state. So, for example, if our agenda contains a single proposition, a precise credence function is some real number between 0 and 1 , whereas an indeterminate credal state is given by a set of numbers, e.g., $\{0.2,0.3\}$, or $[0.2,0.3]$. It remains indefinite which of the credence values in the set it is, but it is, for example, definitely not 0.9 .

This model of belief is closely related to one that is familiar in formal epistemology under the term 'imprecise probabilities', 'indeterminate probabilities', or 'mushy credences'. ${ }^{13}$ It has been proposed for a range of reasons, including being able to represent incomparability as distinct from indifference, distinguishing between lack of evidence and symmetric evidence, allowing for suspension of judgement, and rationalising intuitively rational responses to certain decision problems (Joyce, 2010; Bradley, 2015; Levi, 1978; Jeffrey, 1984). Its interpretation is debated.

To more closely match the application to truth, we might instead identify some particular judgements and ask whether they definitely hold, definitely don't hold, or neither. For example, we might only care about whether one's credence in $\varphi$ is definitely equal to $r$, definitely not equal to $r$, or neither. But the account will be then be very weak. For example, a case like Golf would not get assigned a credence value at all. This thus doesn't respect the fact that your credence should definitely be $\geq 0.3$, which we would get out of the more expressive framework when we look directly at the set of precisifications. Furthermore, this is a difference that might be used in decision making. One might instead then try to be more expansive about the kinds of definite verdicts that are being considered. We might consider whether your credence is definitely $\geq r$ or definitely not $\geq r$. Again, this can be criticised for leaving 
out potentially definite judgements such as that $\varphi$ is more likely than $\psi$, or that $\varphi$ is evidence for $\psi$, or that I'm certain in at least one of $\varphi_{1}, \varphi_{2}, \ldots$.

By focusing on sets of precisifications themselves, however, every definite judgement is encoded. Any $\mathbb{B} \subseteq$ Creds can be thought of as a property of one's credences, for example, $\mathbb{B}=\{c \mid c(\varphi)>c(\psi)\}$ is the property that you think $\varphi$ is more likely than $\psi ; \mathbb{B}=$ $\{c \mid c(\varphi \mid \psi)>c(\varphi)\}$ is the property that you take $\psi$ to be evidence for $\varphi$; and $\mathbb{B}=\left\{c \mid c\left(\varphi_{k}\right)=1\right.$ for some $\left.\left.k\right)\right\}$ is the property that you are certain of at least one of $\varphi_{1}, \varphi_{2}, \ldots$. For any set of precisifications $\mathbb{C}$, we can say whether it definitely satisfies that property, definitely doesn't, or neither, by considering whether $\mathbb{C} \supseteq \mathbb{B}, \mathbb{C} \cap \mathbb{B}=\emptyset$, or neither. Focusing on the set of precisifications, $\mathbb{C}$, itself is equivalent to focusing on definite judgements on all properties, at least when we ignore any differences in definite judgement assignments that don't correspond to differences in resultant precisifications. ${ }^{14}$ And since differences that don't constitute differences in precisifications will not affect the supervaluational jump, considering sets of precisifications themselves is the most general model available for our purposes. Focusing on any particular definite judgements can then be considered as special cases of our general account. Unlike for truth, though, the kinds of triviality issues we face when working with sets of precise credences will often arise for these other models, at least whenever one is interested both in whether a property is definitely satisfied and whether it is definitely not satisfied. (See Section 8.9 for further discussion.)

\subsection{The Jump $\mathcal{R}$ Revising Indeterminate Credence}

\subsection{1 $\mathcal{R}$ Applied to Credences}

We now turn to revision of one's indeterminate credences. Recall our presentation of the supervaluational Kripkean jump for truth as:

$$
\Delta(S)=\operatorname{Def} \circ \mathcal{R}_{\tau} \circ \operatorname{Precs}(S) .
$$

We described this with the following procedure: (i) use Precs to move from a definite verdict assignment to the corresponding set of precisifications; (ii) use the Tarskian revision function, $\tau$, to revise each of these $\left(\mathcal{R}_{\tau}\right)$; and (iii) use Def to move from the resultant set of revised precise interpretations back to the define verdicts assignment.

I suggest that what motivates this definition of $\Delta$ is just the revision of each of the members of the set, $\mathcal{R}_{\tau}$, but since $\Delta$ is defined on the definite verdicts model rather than sets of precisifications, we have to also introduce stages (i) and (iii) to find $\mathcal{R}_{\tau}$ 's treatment of definite verdicts.

In the case of credences, however, we have suggested working directly with a set of precisifications, and want to know how to revise that. So stages (i) and (iii) aren't needed and we might suggest that the analogous 
way to revise a set of precisifications is just to revise each of the precisifications, i.e., just apply stage (ii). We defined $\mathcal{R}_{\tau}$ for the case of truth as $\mathcal{R}_{\tau}(\mathbb{Q}):=\{\tau(Q) \mid Q \in \mathbb{Q}\}$. We now simply present this as a more general definition that can apply to any revision function:

Definition 8.5.1. For a given (fixed) revision function $\rho$,

$$
\mathcal{R}_{\rho}(\mathbb{C}):=\{\rho(c) \mid c \in \mathbb{C}\} .
$$

We will generally drop the subscript as it's typically clear which revision function is used.

This simply takes the collection of revised individuals. It is a very intuitive notion of revision applied to a indeterminate credence, understood as a set of precisifications. It also seems to follow naturally from the supervaluationist idea that what happens on the supervaluational-side supervenes on what happens on the precise side.

Consider Passport, BAD NAvigator or CredLiar. We simply focus on an agenda consisting of the single sentence at stake in each of these scenarios, so credence functions are given by real numbers between 0 and 1 . Supervaluational credences are given by sets of precise credences, so this will be a set of real numbers between 0 and 1 . Consider adopting the set consisting just of the two extremal credences, $\{0,1\}$. Revision of precise credences is spelled out by $\rho_{\text {PASSPORT }}$. In particular, credence 0 recommends adopting credence 1 ; and 1 recommends 0 . When we apply $\mathcal{R}$ to the set $\{0,1\}$ we just revise each member, so we have $\mathcal{R}(\{0,1\})=\{\rho(0), \rho(1)\}=\{1,0\}=\{0,1\}$. The indeterminate credal state $\{0,1\}$ is a fixed point of $\mathcal{R}$. Whilst each precisification is undermining, the set, as a whole, is a non-undermining attitude to adopt in these cases.

We might consider in general saying that an imprecise credal state is non-undermining iff it's a fixed point of $\mathcal{R}$. However, there is a formal issue facing this proposal: $\mathcal{R}$ may not have any (non-trivial) fixed points. Thus, if our notion of underminingness is spelled out just with $\mathcal{R}$, we still might end up with a situation where every credal state, precise or imprecise, is undermining, and thus not a candidate for the rational response to the situation. Ultimately, we will suggest an alternative notion of underminingness which will always allow for a non-undermining response.

\subsection{2 $\mathcal{R}$ Doesn't Always Have a Fixed Point}

Consider the following kind of scenario:

SPRING

You know that you're always overconfident in this type of situation. Except you also know that a credence value of 0 would be wrong. 
Figure 8.1 Illustration of $\rho_{\text {SPRING }}$

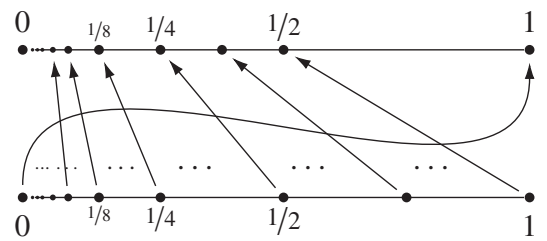

What revision function does this lead to? It will have that $\rho(0)>0$, and for all $x>0, \rho(x)<x$. To say more about it, though, we need further details about this case: 'how overconfident?' 'how wrong?'. In fact, I think natural ways of adding to this story will not guarantee a notion of a particular credence value being recommended, instead it might allow for ties. But for simplicity, this chapter focuses on the case where we have a fully specified revision function. ${ }^{15}$ In fact it doesn't matter how we spell it out, any revision function with these properties leads to a $\mathcal{R}$ which has no fixed points. To work with a concrete example, we suppose that additional details are added to the case so that we obtain the following revision function: ${ }^{16}$

$$
\rho_{\text {SPrING }}(x)= \begin{cases}1 & x=0 \\ \frac{x}{2} & x>0\end{cases}
$$

See Figure 8.1 for an illustration.

As in the cases like PASSPORT, every credence value is undermining. But, unlike in PASSPORT, there is also no indeterminate credal state which is a fixed point of $\mathcal{R}$. Even though $\mathcal{R}$ is monotone, we result in the empty set of precisifications, which is not a legitimate imprecise opinion state.

Proposition 8.5.2. There is no (non-empty) fixed point of $\mathcal{R}_{\text {Spring }}$.

Proof. We will first observe that for any $\mathbb{C}$ and $n \geq 1$, any $x \in \mathcal{R}^{n}(\mathbb{C})$ has $0<x \leq \frac{1}{2^{n-1}}$. (See Figure 8.2.) Recall that $x \in \mathcal{R}^{n}(\mathbb{C})$ iff there is $y \in$ $\mathcal{R}^{n-1}(\mathbb{C})$ with $x=\rho(y)$. So we equivalently need to show that any $x \in$ $\mathcal{R}^{n}(\mathbb{C})$ has $0<\rho(x) \leq 1 / 2^{n}$.

Base case: For any $x>0, \rho(x)=x / 2>0$ (and $\rho(x) \leq 1)$. Also $\rho(0)=$ $1>0$. Thus, any $x \in \mathbb{C} \subseteq[0,1]$ has $0<\rho(x) \leq 1$, as required.

Inductive step: For any $x \in \mathcal{R}^{n}(\mathbb{C}), 0<x \leq 1 / 2^{n-1}$. So $\rho(x)$, which $=x / 2$ has $0<\rho(x) \leq \frac{1 / 2 n-1}{2}=1 / 2^{n}$, as required. 


\begin{tabular}{lccccc}
\hline & $x$ in $\mathcal{R}(\mathbb{Q})$ & $x$ in $\mathcal{R}^{2}(\mathbb{Q})$ & $x$ in $\mathcal{R}^{3}(\mathbb{Q})$ & $x$ in $\mathcal{R}^{4}(\mathbb{Q})$ & $\ldots$ \\
\hline 1 & $\mathbf{x}$ & $\mathbf{x}$ & $\mathbf{x}$ & $\mathbf{x}$ \\
$1 / 2$ & & & $\mathbf{x}$ & $\mathbf{x}$ & $\mathbf{x}$ \\
$1 / 4$ & & & $\mathbf{x}$ & $\mathbf{x}$ \\
$1 / 8$ & & & & & $\mathbf{x}$ \\
$\vdots$ & & & & & \\
0 & & $\mathbf{x}$ & $\mathbf{x}$ & $\mathbf{x}$ & $\mathbf{x}$
\end{tabular}

Figure 8.2 Illustration of $\mathcal{R}$ with SPRING. $x$ can lie only in the gap between the crosses

Now, suppose $\mathbb{C}=\mathcal{R}(\mathbb{C})$. Then $\mathbb{C}=\mathcal{R}^{n}(\mathbb{C})$ for all $n$. So any $x \in \mathbb{C}$ has $x \leq 1 / 2^{n-1}$ for all $n$. But the only such $x$ is 0 , and we also require that $x>0$. So $\mathbb{C}=\varnothing$.

So, this supervaluational jump does not guarantee that undermining credal states can be avoided. So we shouldn't use this alone to characterise underminingness.

\subsection{Supervaluational Kripkean Jump for Truth as It Applies to Sets of Precisifications}

In order to develop a notion of underminingness which will always allow for non-undermining credal states, we first consider how the supervaluational Kripkean jump for truth avoids triviality. This will lead us to consider an alternative jump, closure $\circ \mathcal{R}$. Both jumps, $\mathcal{R}$ and closure $\circ \mathcal{R}$, will be used in the characterisation of underminingness.

\subsection{1 $\mathcal{R}_{\tau}$ Has No Fixed Points}

Some revision functions, such as that for PASSPORT, do lead to fixed points of $\mathcal{R}$, whereas the revision function for SPRING rules them out. What about the Tarskian revision function for truth, $\tau$ ? Does just revising a set of precise interpretations of truth by revising each according to $\tau$ $\left(\mathcal{R}_{\tau}\right)$ have fixed point? It does not. ${ }^{17}$ To show this, we note that the McGee sentence leads to SPRING-style phenomena. The McGee sentence, McGee, is given by:

McGee: Some truth iteration of McGee is not true.

Or, more formally, where

McGee is equivalent to $\neg \forall k>0 \overbrace{T\ulcorner T \ldots\ulcorner T}^{k}\ulcorner\operatorname{McGee}\urcorner\urcorner \ldots\urcorner$. 


\begin{tabular}{|c|c|c|c|c|}
\hline & $Q$ in $\mathcal{R}(\mathbb{Q})$ & $Q$ in $\mathcal{R}^{2}(\mathbb{Q})$ & $Q$ in $\mathcal{R}^{3}(\mathbb{Q})$ & $Q$ in $\mathcal{R}^{4}(\mathbb{Q})$ \\
\hline McGee & \multirow{6}{*}{$n$} & true & true & true \\
\hline$T\ulcorner\mathrm{McGee}\urcorner$ & & \multirow{5}{*}{ 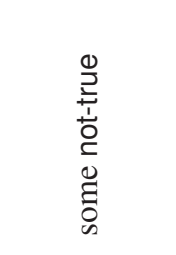 } & true & true \\
\hline$T^{2}\ulcorner\mathrm{McGee}\urcorner$ & & & \multirow{4}{*}{ 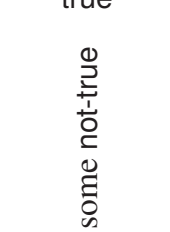 } & true \\
\hline$T^{3}\ulcorner\mathrm{McGee}\urcorner$ & & & & \\
\hline$\vdots$ & & & & tํ \\
\hline & & & & 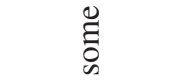 \\
\hline
\end{tabular}

Figure 8.3 Illustration of $\mathcal{R}$ with the McGee sentence.

One can then use this to show:

Proposition 8.6.1 (See also Halbach, 2014, Theorem 14.11). There is no (non-empty) fixed point of $\mathcal{R}_{\tau}$.

Proof. We will first observe that for any $\mathbb{Q}$ and $n \geq 1$, any $Q \in \mathcal{R}^{n}(\mathbb{Q})$ has $\left.T^{i\ulcorner M c G e e}\right\urcorner \in Q$ for all $0 \leq i<n-1$, but also has some $k$ with $T^{k} r$ McGee $\urcorner \notin Q$. (See Figure 8.3.) That is, any $Q \in \mathcal{R}^{n}(\mathbb{Q})$ has $T^{i}\ulcorner\mathrm{McGee}\urcorner \in \tau(Q)$ for all $0 \leq i<n$, but also some $T^{k}\ulcorner\mathrm{McGee}\urcorner \notin \tau(Q)$.

Base case: We just need to show that for any $Q \in \mathbb{Q}$, some $T^{k}\ulcorner$ McGee $\urcorner \notin \tau(Q)$. Since we always have $\varphi \in Q$ iff $T\ulcorner\varphi\urcorner \in \tau(Q)$, if $T^{k}\ulcorner$ McGee $\urcorner \notin Q$, then $T^{k+1}\ulcorner$ McGee $\urcorner \notin \tau(Q)$. So we just need to consider $Q$ where there is no such $k$. For such $Q,(\mathbb{N}, Q) \not \equiv$ McGee, so McGee $\notin \tau(Q)$ giving us our $k=0$.

Inductive step: by our inductive hypothesis we have that any $Q \in \mathcal{R}^{n}(\mathbb{Q})$ has $T^{i}\ulcorner$ McGee $\urcorner \in Q$ for all $0 \leq i<n-1$, but also has some $T^{k}\ulcorner$ McGee $\urcorner$ $\notin Q$. So, since $T\ulcorner\varphi\urcorner \in \tau(Q)$ iff $\varphi \in Q$, we have $T^{i+1}\left\ulcorner M c G e{ }^{\urcorner} \in Q\right.$ for all $0 \leq i<n-1$ and $T^{k+1}\ulcorner\mathrm{McGee}\urcorner \notin Q$. Thus $\left.T^{i\ulcorner M c G e e}\right\urcorner \in \tau(Q)$ for all $1 \leq i<n$ and some $T^{k} r$ McGee $\urcorner \notin \tau(Q)$. Also $(\mathbb{N}, Q) \not$ McGee, so McGee $\in \tau(Q)$ as required for $i=0$.

Now, suppose $\mathbb{Q}=\mathcal{R}(\mathbb{Q})$. Then also $\mathbb{Q}=\mathcal{R}^{n}(\mathbb{Q})$ for all $n$. So any $Q \in \mathbb{Q}$ has $T^{n}\ulcorner$ McGee $\urcorner \in Q$ for all $n$. But also $T^{k}\ulcorner$ McGee $\notin Q$ for some $k$. So $\mathbb{Q}=\varnothing$.

\subsubsection{How $\Delta$ Acts on Sets of Precisifications}

We have thus seen that like for our revision function for the SPRING case, the Tarskian revision function for truth means that revising a set of precisifications by just revising each of the members leads to triviality. However, the usual supervaluational Kripke jump for truth, $\Delta$, does not act on sets of precisifications simply by revising each member of the set (i.e., $\mathcal{R}$ ) instead it acts in accordance with Precs $\circ \operatorname{Def} \circ \mathcal{R}$ : If $\operatorname{Precs}(S)=\mathbb{Q}$, then $\Delta(S)=\operatorname{Def} \circ \mathcal{R}(\mathbb{Q})$, so $\operatorname{Precs}(\Delta(S))=$ Precs $\circ$ Def $\circ \mathcal{R}(\mathbb{Q})$. See Figure 8.4. 


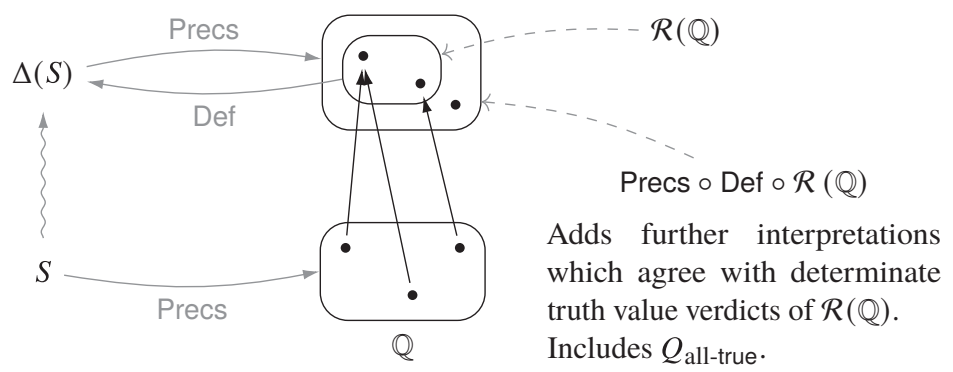

Figure 8.4 Revising a definite verdict assignment and revising the set of precisifications

By applying Precs $\circ$ Def to $\mathcal{R}(\mathbb{Q})$, additional precisifications are added. In particular any additional precisifications that agree with any of the determinate truth value verdicts, i.e., those truth value verdicts which are unanimously agreed on. More carefully: $Q^{*} \in$ Precs $\circ$ $\operatorname{Def}(\mathbb{Q})$ iff for any $\varphi$,

- if $\varphi \in Q$ for all $Q \in \mathbb{Q}$ then $\varphi \in Q^{*}$, and

- if $\varphi \notin Q$ for all $Q \in \mathbb{Q}$ then $\varphi \notin Q$ ".

Any further relationships between sentences do not need to be respected by all $Q^{*}$ in Precs $\circ \operatorname{Def}(\mathbb{Q})$. For example, even if every $Q \in \mathbb{Q}$ has at least one of $\varphi$ or $\psi$ true, if both are indeterminate we can have some $Q^{*} \in \operatorname{Precs} \circ \operatorname{Def}(\mathbb{Q})$ with both $\varphi$ and $\psi$ not true. This means, for example, that even though $\mathcal{R}(\mathbb{Q})$ only contains maximally consistent precise interpretations, Precs $\circ \operatorname{Def} \circ \mathcal{R}(\mathbb{Q})$ can contain inconsistent precise interpretations.

Similarly, each $Q \in \mathcal{R}(\mathbb{Q})$ puts at least one of $T^{k}\ulcorner M c G e e\urcorner$ as not-true. However, since each $T^{k} r$ McGee $\urcorner$ is indeterminate according to $\mathcal{R}(\mathbb{Q})$, so we can find some $Q_{\text {all-true }}$ in Precs $\circ \operatorname{Def} \circ \mathcal{R}(\mathbb{Q})$ which agrees with any determinate truth value verdicts of $\mathcal{R}(\mathbb{Q})$ but which puts all $T^{k}\ulcorner$ McGee $\urcorner$ as true. This is what allows $\Delta$ to have fixed points, where $\mathcal{R}$ does not.

Proposition 8.6.2. There is some $Q_{\text {all-true }}$ in Precs $\circ$ Def $\circ \mathcal{R}($ AllPrecsT $)$ such that we have $T^{k}\ulcorner$ McGee $\urcorner \in Q_{\text {all-true }}$ for all $k$. There is no such $Q_{\text {all- }}$ true in $\mathcal{R}$ (AllPrecsT).

Proof. We argued in 8.6.1 that there is no such $Q_{\text {all-true }}$ in $\mathcal{R}$ (AllPrecsT). We need to show there is some such in Precs $\circ$ Def $\circ \mathcal{R}$ (AllPrecsT).

Consider any $Q_{0} \in$ AllPrecsT. Set $Q_{n}=\tau^{n}\left(Q_{0}\right)$. Note that $Q_{n} \in \mathcal{R}^{n}($ AllPrecsT $)$, and also that $Q_{n} \in \mathcal{R}($ AllPrecs $T)$. 
Define $Q_{\text {all-true }}$ by $\varphi \in Q_{\text {all-true }}$ iff $\varphi$ is stably true in $\left\langle Q_{n}\right\rangle$, that is there is some $k$ with $\varphi \in Q_{n}$ for all $n>k$. Since every $T^{n}\ulcorner$ McGee $\urcorner$ is stable true, it is in $Q_{\text {all-true }}{ }^{18}$

We can then show that $Q_{\text {all-true }} \in$ Precs $\circ$ Def $\circ \mathcal{R}($ AllPrecsT $)$. Note that we have $\left\{Q_{1}, Q_{2}, \ldots\right\} \subseteq \mathcal{R}$ (AllPrecsT). If $\varphi \in Q$ for all $Q \in \mathcal{R}$ (AllPrecsT), then $\varphi \in Q_{n}$ for all $n>0$, so $\varphi \in Q_{\text {all-true }}$. If $\varphi \notin Q$ for all $Q \in \mathcal{R}$ (AllPrecsT), then $\varphi \notin Q_{n}$ for any $n>0$, so $\varphi \notin Q_{\text {all-true }}$. Thus, since $Q_{\text {all-true }}$ agrees with any determinate truth value verdicts of $\mathcal{R}$ (AllPrecsT), it is in Precs $\circ$ Def $\circ \mathcal{R}$ (AllPrecsT) (this is an immediate consequence of the definitions of Precs and Def).

\subsection{An Alternative Jump for Indeterminate Credence}

How can we take these insights and apply them to credence, where we are working directly with the set of precisifications model? When viewed through the lenses of sets of precisifications, the usual supervaluational jump for truth, $\Delta$, adds additional precisifications: it corresponds to Precs $\circ$ Def $\circ \mathcal{R}$ rather than $\mathcal{R}$. We similarly propose an alternative jump for imprecise credences, closure $\circ \mathcal{R}$, which also adds additional precisifications. Which ones? For truth, Precs $\circ$ Def $\circ \mathcal{R}$ adds to $\mathcal{R}$ any precise interpretations which agree on any truth value verdicts that are unanimously agreed on by all $Q \in \mathcal{R}(\mathbb{Q})$. For example, in the case of McGee it adds some $Q_{\text {all-true }}$. For credences, we will instead directly use underlying structure of the real numbers, and take a topological closure.

That is, we will directly consider the jump given by first revising each member of the set, and then taking the closure of the resultant set, where the notion of closure is given as follows:

Definition 8.7.1. $c^{*} \in$ closure $(\mathbb{C})$ iff there is a sequence, $\left\langle c_{\alpha}\right\rangle$, (not necessarily following the revision function) with each $c_{\alpha} \in \mathbb{C}$ and where $\left\langle c_{\alpha}\right\rangle$ converges to $c^{*}$, i.e., where for all $\varphi \in \mathcal{A}$ and for all $\epsilon>0$, there is some $\beta$ such that for all $\alpha>\beta,\left|c_{\alpha}(\varphi)-c^{*}(\varphi)\right|<\epsilon .{ }^{19}$

To see how these definitions work, consider how they apply in the case of SPRING (see Figure 8.5).

$\mathcal{R}$ just revises each member of the set, so recalling the revision function for this case as spelled out in Section 8.5.2, we have

$$
\mathcal{R}\left(\left\{0,1,{ }^{1} /{ }_{2},{ }^{1} / 4, \ldots\right\}\right)=\left\{1,1 / 2,{ }^{1} /{ }_{4},{ }^{1} / 8, \ldots\right\} .
$$

The sequence $\left\langle 1,{ }_{1} /{ }_{2},{ }_{1} /{ }_{4}, \ldots\right\rangle$ converges to 0 , and each member of the sequence is a member of $\mathcal{R}(\{0,1,1 / 2, \ldots\})$. So when we take the closure of this set we will add 0 , resulting in:

$$
\begin{aligned}
\text { closure } \circ \mathcal{R}\left(\left\{0,1,{ }^{1} /{ }_{2},{ }^{1} /{ }_{4}, \ldots\right\}\right) & =\operatorname{closure}\left(\left\{1,{ }^{1} /{ }_{2},{ }^{1} /{ }_{4},{ }^{1} / 8, \ldots\right\}\right) \\
& =\left\{0,1,{ }^{1} /{ }_{2},{ }^{1} /{ }_{4}, 1 / 8, \ldots\right\},
\end{aligned}
$$




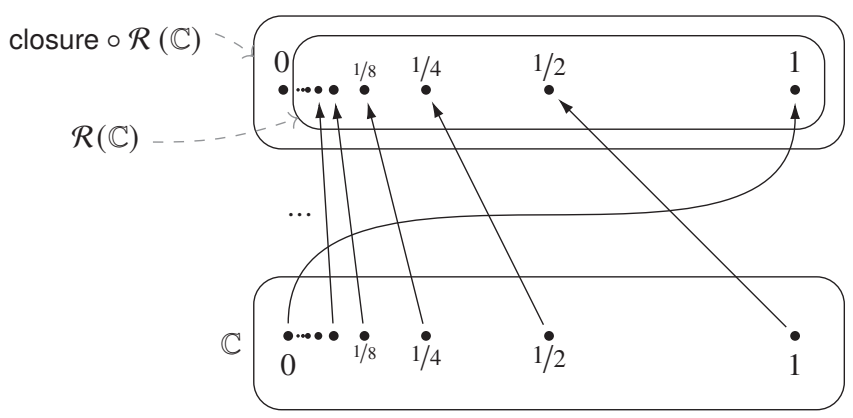

Figure 8.5 By including the additional credence, 0, we find a fixed point in the case of SPRING

This set is a fixed point of closure $\circ \mathcal{R}$ in the SPRING case.

In order to show our general result that closure $\circ \mathcal{R}$ always has fixed points, we need to say a bit more about this notion of closure.

A space, in this case Creds $_{\mathcal{A}}$, with a notion of closure gives us a topology. ${ }^{20}$ In fact, the notion of closure defined in Definition 8.7.1 gives us the so-called topology of pointwise convergence, with the underlying topology on the real numbers being the standard one. There is an important property of this topology: it is compact. This is the property that allows us to show that closure $\circ \mathcal{R}$ has fixed points.

We can now move to defining compactness (which we state in the form that we need for our main result ${ }^{21}$ ).

Definttion 8.7.2. $\mathbb{C}$ is closed if $\mathbb{C}=$ closure $(\mathbb{C})$.

Definition 8.7.3. A space with a notion of closure is compact iff whenever $\mathcal{C}$ is a collection of closed sets which has the finite intersection property,

i.e., for any finite sub-collection $C_{1}, \ldots, C_{k} \in \mathcal{C}, C_{1} \cap \ldots \cap C_{k} \neq \varnothing$ then $\cap \mathcal{C} \neq \varnothing$.

Proposition 8.7.4. Creds $_{\mathcal{A}}$ (with the specified notion of closure) is compact.

Proof Idea. Note that $[0,1]$ is compact as it is a closed and bounded subset of $\mathbb{R}$. Tychonoff's theorem (see, e.g., Willard, 1970, Theorem 17.8) says that the product of compact spaces is compact. We gave the notion of closure which corresponds to the topology of pointwise convergence, which is just the product topology $[0,1]^{\mathcal{A}}$, and is therefore compact. 
There are some other properties that are equivalent to compactness: that every convergent sequence has a limit point, or that every sequence whatsoever has a cluster point. ${ }^{22}$ If we call a collection of sets consistent if it has some common member, i.e., has non-empty intersection, then we can describe this as: any collection of closed sets which is finitely consistent is consistent. Since we are looking for fixed points of closure $\circ \mathcal{R}$ rather than $\mathcal{R}$ we can focus just on closed properties, as is done in the topological definition of compactness.

This allows us to show our main result:

Theorem 8.7.5. For any $\rho$, there is a non-empty fixed point of closure $\circ \mathcal{R}$.

Proof. Define a sequence

- $\mathbb{C}_{0}:=$ Creds $_{\mathcal{A}}$,

- $\mathbb{C}_{\alpha+1}:=$ closure $\circ \mathcal{R}\left(\mathbb{C}_{\alpha}\right)$,

- $\mathbb{C}_{\mu}:=\bigcap_{\alpha<\mu} \mathbb{C}_{\alpha}$.

closure $\circ \mathcal{R}$ is monotone, that is:

Sublemma 8.7.5.1. If $\mathbb{C} \supseteq \mathbb{C}^{\prime}$ then closure $\circ \mathcal{R}(\mathbb{C}) \supseteq$ closure $\circ \mathcal{R}\left(\mathbb{C}^{\prime}\right)$.

Proof. It is easy to observe that $\mathcal{R}$ is monotone, that is, if $\mathbb{C} \supseteq \mathbb{C}^{\prime}$ then $\mathcal{R}(\mathbb{C}) \supseteq \mathcal{R}\left(\mathbb{C}^{\prime}\right)$.

Also, closure is monotone: Suppose $\mathbb{C} \supseteq \mathbb{C}^{\prime}$. For any, $c^{*} \in$ closure $\left(\mathbb{C}^{\prime}\right)$, there is a sequence $\left\langle c_{\alpha}\right\rangle$ in $\mathbb{C}^{\prime}$ which convergs to $c^{*}$. This sequence is also a sequence in any $\mathbb{C} \supseteq \mathbb{C}^{\prime}$. So $c^{*} \in \operatorname{closure}(\mathbb{C})$.

And thus, closure $\circ \mathcal{R}$, which is the result of composing these, is also monotone.

So, by starting with $\mathbb{C}_{0}=$ Creds $_{\mathcal{A}}$, where we have $\mathbb{C}_{0} \supseteq$ closure $\circ \mathcal{R}\left(\mathbb{C}_{0}\right)$, we have that for $\alpha<\beta, \mathbb{C}_{\alpha} \supseteq \mathbb{C}_{\beta}$; and there must be a (possibly empty) fixed point of closure $\circ \mathcal{R}$.

We need to check that this fixed point is non-empty, which we do by induction. ${ }^{23}$

- Base case: $\mathbb{C}_{0}=$ Creds $_{\mathcal{A}} \neq \varnothing$.

- Successor case: For any $c \in \mathbb{C}_{a}, \quad \rho(c) \in \mathcal{R}\left(\mathbb{C}_{\alpha}\right)$, and since closure $\left(\mathbb{C}^{\prime}\right) \supseteq \mathbb{C}^{\prime}$ for any $\mathbb{C}^{\prime}$ (as the constant sequence $\left\langle c^{\prime}, c^{\prime}, \ldots\right\rangle$ converges to $c^{\prime}$ ), also $\rho(c) \in \operatorname{closure}\left(\mathcal{R}\left(\mathbb{C}_{\alpha}\right)\right)=\mathbb{C}_{\alpha+1}$.

- Limit case: Suppose each $\mathbb{C}_{\alpha} \neq \varnothing$ for $\alpha<\mu$. $\left\{\mathbb{C}_{\alpha} \mid \alpha<\mu\right\}$ is a collection of closed subsets of Creds. For $\alpha<\beta, \mathbb{C}_{\alpha} \supseteq \mathbb{C}_{\beta}$, so any finite subcollection has a non-empty intersection. Thus, by definition 8.7.3, $\mathbb{C}_{\mu} \neq \varnothing$. 
We will now spell out a notion of underminingness applied to imprecise credences, and use this result to show that there are always some non-undermining credal states.

\subsection{Characterising Underminingness}

We had originally considered suggesting that an imprecise credal state is non-undermining iff it is a fixed point of $\mathcal{R}$. But with this definition, in the case of SPRING, not only are all precise credence functions undermining, also all the imprecise credences are too. However, we have now considered also taking the closure of $\mathcal{R}$, and seen that closure $\circ \mathcal{R}$ will always have fixed point imprecise credences.

We propose to characterise undermining imprecise credal states by: ${ }^{24}$

DefintTIOn 8.8.1. $\mathbb{C}$ is non-undermining iff $\mathcal{R}(\mathbb{C}) \subseteq \mathbb{C} \subseteq$ closure $(\mathcal{R}(\mathbb{C}))$.

This says that every $c \in \mathbb{C}$ should have its recommended credence in the set, i.e., $\mathcal{R}(\mathbb{C}) \subseteq \mathbb{C}$, and that every credence function in the set should either be recommended by some member of the set or be the limit of a sequence of such recommended functions, i.e., $\mathbb{C} \subseteq$ $\operatorname{closure}(\mathcal{R}(\mathbb{C}))$.

This definition allows that any $\mathbb{C}$ which is a fixed point of $\mathcal{R}$ is nonundermining. ${ }^{25}$ So are any fixed points of closure $\circ \mathcal{R}$, and thus by Theorem 8.7.5 there is always some non-undermining credal state. A state can also be non-undermining without being a fixed point of either of these if it contain some but not all members of the closure. ${ }^{26}$

What credal states are non-undermining in the cases mentioned? (Most of these were introduced in Section 8.3.)

- For the Passport case, $\{0,1\}$ is the only credal state which is nonundermining. The same revision function is used for BAD NAvigATOR and CredLiar, so the same holds for these cases too.

- Golf is similar, and the only non-undermining credal state is $\{0.495$, $0.505\}$.

- For normal cases, like RaIn, where $\rho(c)=c$ for all $c$, every imprecise credal state, $\mathbb{C}$, is a fixed point of $\mathcal{R}$, and thus is non-undermining. Note that if we required states to be fixed points of closure $\circ \mathcal{R}$, then a set which is not closed, such as $(0.2,0.8)=\{x \mid 0.2<x<0.8\}$ would be undermining as it doesn't contain its limit points of 0.2 and 0.8 . But we would like to say that it is non-undermining, and our definition allows this.

- Since the revision function of LeAP is identical to that of RaIN, it too says that any $\mathbb{C}$ is non-undermining.

- For an extremal version of LEAP where $\rho(x)=1$ if $x \geq 0.5$ and $\rho(x)=0$ if $x<0.5$, the non-undermining options are 0,1 and $\{0,1\} .{ }^{27}$ 
- For Promotion, the precise credence 0.5 is non-undermining. But so is any imprecise credence with $x \in \mathbb{C}$ iff $1-x \in \mathbb{C}$, e.g., $\{0.2,0.8\}$, or $(0.2,0.8) .^{28}$

- For Spring, $\left\{0,1,1 / 2,{ }^{1} / 4, \ldots\right\}$ is a fixed point of closure $\circ \mathcal{R}$; it is the only non-undermining state.

\subsection{Other Applications and General Considerations}

We have here considered the notions of truth and rational credence. But the considerations and construction we give here is very general. It could fruitfully apply to a whole range of target domains, for example: reference or satisfaction; membership or exemplification; necessity or knowledge; or decision theoretic or game theoretic rationality. ${ }^{29}$ All one needs in order to apply it to a target domain is to specify the collection of all potential precisifications, AllPrecs, and how to revise each of them, i.e., specify a revision function, $\rho:$ AllPrecs $\rightarrow$ AllPrecs. The revision function should be such that one would like to find fixed points of it, though this may not be possible. This can be done for all the domains mentioned.

For truth, AllPrecs was given by $\mathcal{P}\left(\right.$ Sent $\left._{T}\right)$ and the revision function was spelled out with Tarskian truth revision jump, $\tau$. But we could also consider variations of this setup, for example, we might consider restricting to just certain kinds of precise interpretations, for example those that are maximally consistent.

For credences, AllPrecs was given by Creds $_{\mathcal{A}}=[0,1]^{\mathcal{A}}$ and we just took the revision function to be (externally) given. We could also modify this setup. We could restrict it to just the functions that are (finitely additive) probability functions. We could also consider credence, or probability, as spelled out over possible world structures. (See Section 8.3.2 for a brief description of this and the associated revision function.) We can thus obtain a supervaluational variant of a Kripkean account of probability paralleling the strong Kleene version developed in Campbell-Moore (2015), or a probabilistic variant of the supervaluational Kripkean account of necessity as in Nicolai (2018). ${ }^{30}$ There are some advantages of the supervaluational approach over the strong Kleene one, especially as understood as giving sets of credal-evaluation-functions. For example, we can immediately read off a whole range of definite facts, for example about conditional probability, whereas it's not immediately clear how to consider conditional probability in a strong Kleene framework. We might also consider joint theories of credence and truth.

Once one has a collection of potential precisifications, we can then consider the indeterminate variant of one's target notion to be given by sets of precisifications. And we can define an operator $\mathcal{R}$ which applies to a set of precisifications just by revising each precise 
interpretation in accordance with the specified revision function. Whilst very natural, this will also typically not guarantee fixed points.

Having further investigated the usual supervaluational account for truth as it applies to sets of precisifications, we proposed allowing one to add additional precisifications which are not individually recommended, but are in the closure of the set of recommended precisifications. To apply this in general, one also needs to define an appropriate notion of closure, which should be monotone and increasing. Many domains come along with natural notions of closure. ${ }^{31}$ Often this will be compact, as for example our topology on Creds was. There are some limitations, for example, if we restrict the precise interpretations of truth to those that are $\omega$-consistent, or the credence functions to those that are countably-additive, compactness is lost. Whenever the space it is compact, fixed points of closure $\circ \mathcal{R}$ can be found.

We then proposed a notion of when indeterminate credal states are non-undermining: when $\mathcal{R}(\mathbb{C}) \subseteq \mathbb{C} \subseteq \operatorname{closure}(\mathcal{R}(\mathbb{C}))$. And we observed that since closure $\circ \mathcal{R}$ has non-trivial fixed points, there are always some non-undermining credal states. The analogous definition could be applied to other domains and further investigated. Or one might explicitly focus on closure $\circ \mathcal{R} .^{32}$

Our main focus has been on sets of precisifications, but one might be interested some independently specified supervaluational models. For example, just focusing on certain definite judgements as is usually done for truth. Again, the general account we have developed here can immediately apply. To apply it, one should spell out operators analogous to Precs and Def, which we used for truth, that allow us to associate sets of precisifications with one's independently specified models. One can then consider two jumps on these supervaluational models, one which tracks the action of $\mathcal{R}$ on one's supervaluational models (Def $\circ \mathcal{R} \circ$ Precs), and the other that tracks the action of closure $\circ \mathcal{R}$ (Def $\circ$ closure $\circ \mathcal{R} \circ$ Precs). Usually, the jump of one's supervaluational models corresponding to closure $\circ \mathcal{R}$ will obtain fixed points whereas that corresponding to $\mathcal{R}$ often does not.

However sometimes the action of $\mathcal{R}$ on one's supervaluational models does lead to fixed points even though $\mathcal{R}$ itself does not. This is what we observed in the case of truth where the action of $\mathcal{R}$ on definite verdict assignments was given by $\Delta$ and had non-trivial fixed points even though $\mathcal{R}$ did not. This is because in this special case, the jumps $\mathcal{R}$ and closure $\circ \mathcal{R}$ are identical insofar as they act on definite verdict assignments. The formal reason for this is that any $Q^{*} \in \operatorname{closure}(\mathbb{Q})$ is in Precs $\circ \operatorname{Def}(\mathbb{Q})$.

But this typically won't be the case, especially for a notion like credence. For example, if we work with definite judgements regarding one's credences, $\mathcal{R}$ and closure $\circ \mathcal{R}$ will act differently. Consider focusing on definite judgements as to whether one's credence in $\varphi$ is $>r$ or not. In the case of 
SPRING, for example, every $c \in \mathcal{R}$ (Creds) has $c$ (SPRING) $>0$, so this is a definite judgement, but when we consider closure $\circ \mathcal{R}$ (Creds) we find that $c(\varphi)>0$ is no longer definitely satisfied as some member of the closure has $c(\varphi)=0$. This means that even once we consider definite judgements in the case of credences, we have to work with the jump analogous to closure $\circ \mathcal{R}$ rather than $\mathcal{R}$ to ensure there are fixed points.

It would not help to focus on definite judgements regarding different properties. For example, regarding whether one's credence is $\geq r$, or equal to $r$, at least if one is interested both in whether it definitely holds and whether it definitely doesn't hold. This is because for either the positive or negative component, taking a closure can make a difference, and thus the jumps corresponding to $\mathcal{R}$ and closure $\circ \mathcal{R}$ will differ, and it's only closure $\circ \mathcal{R}$ that will guarantee fixed points. Formally, this is because, for the case of credences, a set and its complement won't both be closed (unless one of them is empty). It was a special feature of truthvalues (they're discrete) that meant that focussing on definite truth value verdicts allowed triviality to be avoided. ${ }^{33}$

There are still ways to avoid such worries. Consider modelling one's supervaluational credences with a partial function, where to to some sentences you do not form any attitude whatsoever, and to the others, you assign normal precise values. This is essentially caring about whether one's credence is definitely equal to $r$ without caring about whether it is definitely not equal to $r$. Since taking a closure of a set cannot reject any definite credence value assignments, $\mathcal{R}$ and closure $\circ \mathcal{R}$ act identically on these definite credence value assignments, and thus there will be fixed points of the jump associated with $\mathcal{R}$ (as there are fixed points of the jump associated with closure $\circ \mathcal{R}$ ). This will be the case whenever we only consider positive definite judgements on closed properties. But one very legitimately might be interested in other properties, such as whether one's credence is definitely $>r$, or if it's definitely not equal to $r$, in which case closure $\circ \mathcal{R}$ needs to be considered.

In summary: We have proposed an account of underminingness applying to indeterminate credal states where there are always nonundermining indeterminate credal states even in the face of certain scenarios which have recently been considered as a challenge to rationality, and which bear a close relationship to the liar paradox. Along the way, we have obtained a deeper understanding of the supervaluational Kripkean account of truth, especially as it applies to sets of precisifications, and offered a very general account that could be applied to a whole range of target domains.

\section{Acknowledgments}

Thank you very much to Johannes Stern and Carlo Nicolai for insightful conversations and detailed feedback on this chapter. Variants of this talk 
have been in numerous conferences and seminars, I'm grateful for the organisers and the many helpful questions and conversations. My research was supported by a Leverhulme Trust Research Fellowship.

\section{Notes}

1. Recent discussion of such cases was initiated by Caie (2013) and Greaves (2013). However they both consider rationality considerations to apply to these cases in a consequentialist manner, which is not the way I am working with them. Instead, I am following Konek and Levinstein (2019) and Joyce (2018). See also Carr (2017) and Pettigrew (2018) for further discussion.

2. We have chosen to follow McGee $(1989,1990)$ in thinking about this as definite truth rather than a partial interpretation of truth as Kripke (1975) did. All the formal work would equally well apply to the partial interpretations picture.

3. Whilst we officially allow that sentences may be both in $S^{+}$and $S^{-}$, such definite verdict assignments will have no precisifications, and thus will be trivial.

4. It would not affect our account if we took them to be propositions understood in a different way, e.g., they could be sets of possible worlds.

5. We may need to ensure that $\mathcal{A}$ contains all relevant sentences to obtain the revision notion; that is, the rationally recommended credence value of a sentence in $\mathcal{A}$ is settled by a hypothesis about the credence value of sentences in $\mathcal{A}$. See the notion of self-ref agenda from Campbell-Moore (2016, section 6.2).

6. See, e.g., Pettigrew (2016, Definition 1.0.1).

7. To see that this is not compact, observe that the limit of a convergent sequence of countably additive probabilities might be merely finitely additive. This is not the case for finite additivity.

8. In fact, many situations will not give rise to a recommendation function. Instead, maybe there are ties. Our whole account can be expanded to deal with ties, see note 23 . However, this would complicate the presentation and the parallel to the truth case, so I assume that the notion of revision is functional.

9. In fact, one might want to specify $\rho$ so that we only have $\rho(c)=c$ if $c$ satisfies further principles of rationality, for example the Principal Principle.

10. For arguments for this in our "unusual" cases see especially (Joyce, 2018). For this argument in "safe" cases, see discussions of immodesty, e.g., Joyce (2009) and Lewis (1971). Someone like Pettigrew (2018) who disagrees with Joyce on these "unusual" cases might be thought of as agreeing with the idea that one's credence should be a fixed point of $\rho$, but instead works with an implementation of $\rho$ which is consequentialist. It turns out then that $\rho$ does not depend on the input value at all, and thus it always has a fixed point.

11. Joyce in fact proposes that $\rho(c)$ is the function that minimises expected inaccuracy, given that $c$ is chosen (Joyce, 2018, p. 257), but this will typically simply be the $c$ thus conditionalised.

12. See Campbell-Moore (2016, Section 5.3). In Section 4.1, I also extended this to the imprecise setting and considered $\mathcal{R}$. However, it is there claimed that $\mathcal{R}$ will guarantee fixed points, but these might be empty due to considerations in this chapter.

13. There is in fact a whole range of models of belief that are discussed. Many of these are weaker than arbitrary sets of probabilities, e.g., upper-lower probability models. However, especially under the term "imprecise probabilities", some models are stronger as they can encode opinions that would only be captured by non-Archimedean probability functions. Campbell-Moore and Konek (2019) present a model of belief that is more general than all 
those considered in the imprecise probability literature, and where considerations such as those in this chapter might also be able to be applied. Moreover, some of the issues we find of ensuring fixed points may be more easily avoided and will be presented in a future article.

14. For example if two definite judgement assignments both have no precisifications, they are treated as identical.

15. See also note 23.

16. Our choice of $\rho(0)=1$ is an extreme way to spell out the details of the story: if you assign credence 0 , you think it's definitely true. We have made this choice as it is then parallel the McGee sentence which we will discuss in Section 8.6.1.

17. When we conceive of it as apply to the whole language, which includes, for example, the McGee sentence. It does have fixed points when applied, e.g., just to Liar.

18. This style of argument can directly be used to show that closure $\circ \mathcal{R}$ has nontrivial fixed points, see note 22 .

19. If $\mathcal{A}$ is countable, we just need to look at $\omega$-length sequences.

20. See Willard (1970, Theorem 3.7). To apply these considerations in general one does not need the notion of closure to satisfy all the usual properties, it suffices to assume that closure is monotone and increasing.

21. This is equivalent to the usual definition of compactness, see Willard (1970, Theorem 17.4).

22. See, e.g., Willard (1970, Theorem 17.4). See also notes 22 and 25 for use of this alternative picture and a comment that it then offers a close relationship to the revision theory.

23. Alternative arguments are possible: Firstly, we could observe that what compactness shows us is that the non-empty closed subsets of $\mathbb{C}$ forms a ccpo in the sense of Visser (1984). And since we can restrict attention to the closed subsets for the purposes of closure $\circ \mathcal{R}$, there must be a fixed point. Secondly, we can define a revision sequence: $c_{0} \in \mathrm{Creds}_{\mathcal{A}}, c_{\alpha+1}=\rho\left(c_{\alpha}\right)$, and let $c_{\mu}$ be a cluster point of the preceding sequence (in Campbell-Moore, 2019 we proposed using this as the limit criterion in the revision theory), and observe that $c_{\alpha} \in \mathbb{C}_{\alpha}$. This relies on the ability to always find a cluster point, which is equivalent to compactness.

24. To extend this to the case where $\rho$ doesn't pick out a unique credence function but can allow for ties, we will say that $\mathbb{C}$ is non-undermining iff each $c \in$ $\mathbb{C}$ has at least one of their maximally recommended credences in $\mathbb{C}$, and everything in $\mathbb{C}$ is in the closure of recommended credences.

25. This is a key reason to give our definition rather than saying that it has to be a fixed point of closure $\circ \mathcal{R}$. I would like to say the further thing that they are preferable: if they exist then they are required, but the criterion does not do this. To account for this intuition, we might also define a notion of recommendation for imprecise credences as: $\mathbb{C}$ recommends $\mathcal{R}(\mathbb{C})$ and say that ideally one's credal state should be self-recommending, but if it cannot be, it should at least be non-undermining.

26. A further advantage of this definition is it leads to a nice relationship with the revision theory of Gupta and Belnap (1993). If one has a revision sequence, following $\rho$ as the revision step and using the limit criterion that the limit be a cluster point of the preceding sequence, then the collection of members of the looping part of the sequence is non-undermining. So are any unions of such revision loops. (However, there are non-undermining states which are not unions of such revision loops.)

27. This could be described as a "truth-teller" variant of CredLiar. 
28. In fact we can find further non-undermining states such as $(0.2,0.8)$.

29. The first three of these collections of examples are taken from Gupta and Belnap (1993, Section 7.2), game theory is another place where revision theory has been applied (Bruni and Sillari, 2018), and decision theoretic cases such as Death in Damascus (Gibbard and Harper, 1978) where every action is undermining would be another natural application.

30. Nicolai, along with all the other work on predicate approaches to modality, does not consider sets of precisifications but rather, in our terms, whether a sentence is definitely necessary (at world $w$ ) or definitely not necessary (at $w$ ).

31. Often one has an underlying domain of "values", such as $\{$ true, not-true $\}$ or $[0,1]$, and the space of precisifications is the collection of functions from some other objects such as sentences, or pairs of worlds and sentences, to these values. One then just needs to impose a topology (or notion of closure) on the underlying values and then can use the topology of pointwise convergence (the product topology) to obtain a notion of closure on the collection of all possible precisifications. By Tychonoff's theorem this will be compact so long as the topology on the underlying values is compact (as \{true, not-true\} or $[0,1]$ is). One might also consider only special kinds of functions (for example those that are maximally consistent), in which case one should also check that the set of all such functions is closed in the full function space. See a text on general topology such as Willard (1970). See also Campbell-Moore (2019) where we used this topology for truth and probability to take limits in the revision theory.

One could also work with alternatives, such as simply defining closure $(\mathbb{Q})$ as Precs $\circ \operatorname{Def}(\mathbb{Q})$ itself. This would suffice, but this will generally be more permissive than closure defined by the topology of pointwise convergence. An interesting exception is if we restrict AllPrecsT to those that are maximally consistent where then these are identical.

32. For credences, this seems unmotivated, but perhaps it is appropriate for other domains.

33. As both the property of a sentence being true and being not-true are closed properties.

\section{References}

Bradley, S. (2015). Imprecise probabilities. In Zalta, E. N., editor, The Stanford Encyclopedia of Philosophy, Summer 2015 edition.

Bruni, R. and Sillari, G. (2018). A rational way of playing: Revision theory for strategic interaction. Journal of Philosophical Logic, 47(3): 419-448.

Caie, M. (2013). Rational probabilistic incoherence. Philosophical Review, 122 (4): 527-575.

Campbell-Moore, C. (2015). How to express self-referential probability. A Kripkean proposal. The Review of Symbolic Logic, 8: 680-704.

Campbell-Moore, C. (2016). Self-Referential Probability. PhD thesis, LudwigMaximilians-Universität, München.

Campbell-Moore, C. (2019). Limits in the revision theory. Journal of Philosophical Logic, 48(1): 11-35.

Campbell-Moore, C. and Konek, J. (2019). Believing probabilistic contents: On the expressive power and coherence of sets of sets of probabilities. Analysis, 80 (2): 316-331.

Carr, J. R. (2017). Epistemic utility theory and the aim of belief. Philosophy and Phenomenological Research, 95(3): 511-534. 
Egan, A. and Elga, A. (2005). I can't believe I'm stupid. Philosophical Perspectives, 19(1): 77-93.

Gibbard, A. and Harper, W. L. (1978). Counterfactuals and two kinds of expected utility. In Ifs, pages 153-190. Springer.

Greaves, H. (2013). Epistemic decision theory. Mind, 122: 915-952.

Gupta, A. and Belnap, N. D. (1993). The Revision Theory of Truth. MIT Press.

Halbach, V. (2014). Axiomatic Theories of Truth. Cambridge University Press, Revised edition.

Halbach, V., Leitgeb, H., and Welch, P. (2003). Possible-worlds semantics for modal notions conceived as predicates. Journal of Philosophical Logic, 32: 179-222.

Halbach, V. and Welch, P. (2009). Necessities and necessary truths: A prolegomenon to the use of modal logic in the analysis of intensional notions. Mind, 118 (469): 71-100.

James, W. (1897). The will to believe. In The Will to Believe and Other Essays in Popular Philosophy, pages 1-15. New York: Longmans, Green, and Co.

Jeffrey, R. (1984). Bayesianism with a human face. In Earman, John, editor, Testing Scientific Theories. University of Minnesota Press, pages 133-156.

Joyce, J. M. (2009). Accuracy and coherence: Prospects for an alethic epistemology of partial belief. In Degrees of Belief, pages 263-297. Springer.

Joyce, J. M. (2010). A defense of imprecise credences in inference and decision making. Philosophical Perspectives, 24: 281-323.

Joyce, J. M. (2018). Accuracy, ratification, and the scope of epistemic. In Ahlstrom-Vij, K. and Dunn, J., editors, Epistemic Consequentialism, chapter 10. Oxford University Press.

Konek, J. and Levinstein, B. A. (2019). The foundations of epistemic decision theory. Mind, 128(509): 69-107.

Kripke, S. (1975). Outline of a theory of truth. The Journal of Philosophy, 72 (19): 690-716.

Levi, I. (1978). On indeterminate probabilities. In Foundations and Applications of Decision Theory, pages 233-261. Springer.

Lewis, D. (1971). Immodest inductive methods. Philosophy of Science, 38: 54-63.

McGee, V. (1989). Applying Kripke's theory of truth. The Journal of Philosophy, 86(10): 530-539.

McGee, V. (1990). Truth, Vagueness, and Paradox: An Essay on the Logic of Truth. Hackett Publishing.

Nicolai, C. (2018). Necessary truths and supervaluations. From Arithmetic to Metaphysics, 73: 309.

Pettigrew, R. (2016). Accuracy and the Laws of Credence. Oxford University Press.

Pettigrew, R. (2018). Making things right: The true consequences of decision theory in epistemology. In Ahlstrom-Vij, K. and Dunn, J., editors, Epistemic Consequentialism, chapter 9. Oxford University Press.

Stern, J. (2015). Toward Predicate Approaches to Modality, volume 44 of Trends in Logic. Springer.

Visser, A. (1984). Four valued semantics and the liar. Journal of Philosophical Logic, 13(2): 181-212.

Willard, S. (1970). General Topology. Courier Corporation. 